\title{
Low-Sensitivity Digital-Filter Structures which are Amenable to Error-Spectrum Shaping
}

\author{
PAULO S. R. DINIZ, MEMBER, IEEE, AND ANDREAS ANTONIOU, FELLOW, IEEE
}

\begin{abstract}
A systematic and exhaustive procedure is used to generate a class of new low-sensitivity second-order digital-filter structures which are amenable to error-spectrum shaping (ESS). Collectively, these structures can realize any stable second-order transfer function. For every transfer function, a choice of at least two structures is available but, through a sensitivity analysis, the optimum one can always be identified. The paper concludes with sensitivity and roundoff-noise comparisons which show that the new structures are usually superior relative to corresponding direct canonic structures and also relative to the state-space section-optimal structure.
\end{abstract}

\section{INTRODUCTION}

$\mathbf{R}$ OUNDOFF noise can be reduced in recursive digital filters by increasing the wordlength [1], by choosing the structure appropriately [2], [3], or by applying errorspectrum shaping (ESS) [4]-[8].

ESS is a quantization technique which involves the generation of an error signal and the application of local feedback for the purpose of forcing zeros in the power spectral density of the output noise. The technique can be implemented by incorporating a quantizer and a corresponding substructure between the output and input of each and every adder whose inputs include at least one nontrivial product (a signal multiplied by a noninteger constant). Therefore, the application of ESS entails an increase in the complexity of hardware. Furthermore, the complexity of hardware increases as the number of adders of the type described is increased and, consequently, the application of this technique has been restricted to direct cascade realizations in the past [4]-[8].

The application of ESS to direct realizations tends to bring about a dramatic reduction in the output roundoff noise [4]-[8]. Unfortunately, however, the sensitivity to coefficient quantization is not affected by ESS and, as is well known, it can be large, in particular if the poles of the transfer function are close to the unit circle of the $z$ plane [9], [10].

In this paper, a systematic procedure is described which can be used for the generation of low-sensitivity digital-filter structures which are amenable to ESS. The procedure is used to generate two sets -of structures which include several new structures as well as some known structures

Manuscript received June 7, 1984; revised February 8, 1985

P. S. R. Diniz is with the Department of Electrical Engineering, Universidade Federal do Rio de Janeiro, 21944 Rio de Janeiro, Brazil.

A. Antoniou is with the Department of Electrical Engineering, University of Victoria, Victoria, B.C., Canada V8W 2 Y2.

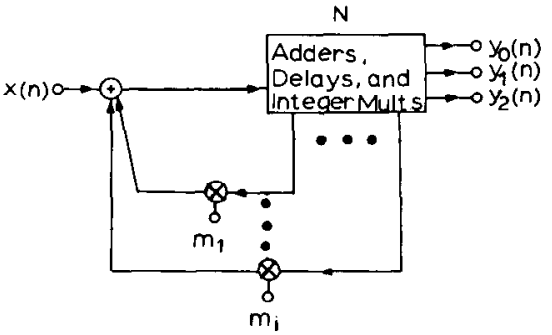

Fig. 1. General structure which is amenable to ESS.

like those of Agarwal and Burrus [9], and Nishimura, Hirano, and Pal [10]. The emphasis is placed on generating second-order structures which can be used in cascade or in parallel for the realization of high-order transfer functions.

\section{Synthesis Procedure}

The application of ESS in a second-order structure is economically attractive only if product quantization can be achieved by using no more than one quantizer. Consequently, a second-order structure is amenable to ESS only if all nontrivial signal-coefficient products in the structure are inputs to only one adder.

A general second-order structure can be constructed as depicted in Fig. 1 where $y_{0}(n), y_{1}(n)$, and $y_{2}(n)$ are internal node signals which will be used later for the placement of zeros. If coefficients $m_{i}$ are assumed to be noninteger constants and substructure $N$ is assumed to be free of noninteger multipliers, a general second-order structure is obtained which is amenable to ESS. By applying a systematic and exhaustive search to the general structure of Fig. 1, all possible low-sensitivity structures can be generated and appropriate design formulas can be deduced.

In this section, the above approach is used for the realization of all-pole transfer functions. The realization of biquadratic transfer functions is accomplished through zero-placement techniques and is considered in Section III.

The simplest structure that can realize any second-order allpole transfer function must have three nodes [11], where each node may represent a distribution or a summation point. The most general structure of this class will have forward and reverse paths between any two of the three nodes and, further, either the forward or reverse path in each case must correspond to a unit delay in order to avoid delay-free loops. On this basis, the simplest form of the general structure of Fig. 1 is obtained, as shown in Fig. 2, 


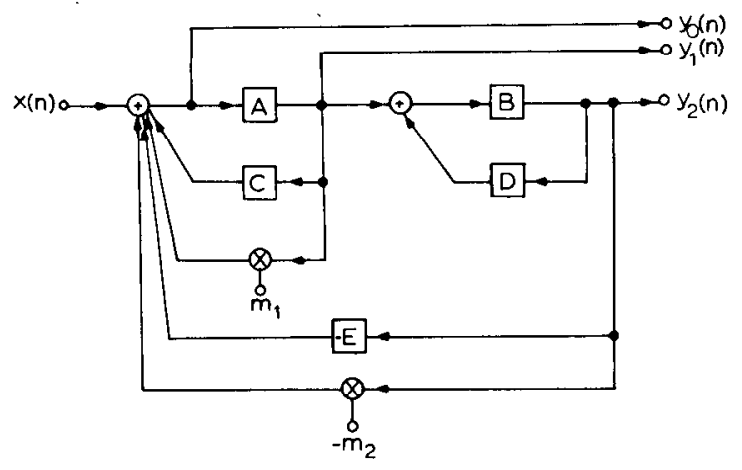

Fig. 2. Second-order structure of low complexity which is amenable to ESS

where branches $A, B, C, D$, and $E$ represent unit delays or integer multipliers whose multiplier constants are restricted to $0, \pm 1, \pm 2$. Evidently, a forward path has not been included between the input adder and node $y_{2}(n)$ since this would necessitate a third unit delay.

The structure of Fig. 2 realizes the characteristic polynomial .

$$
\begin{aligned}
D(z)= & \left(1-B D-A C-m_{1} A+A B E+m_{2} A B\right. \\
& \left.+A B C D+m_{1} A B D\right) z^{2} \\
= & z^{2}+\alpha_{1} z+\alpha_{2} \\
= & \left(z-r e^{j \omega_{0}}\right)\left(z-r e^{-j \omega_{0}}\right)
\end{aligned}
$$

where

$$
r=\sqrt{\alpha_{2}}, \quad \omega_{0}=\cos ^{-1}\left(-\alpha_{1} / 2 \sqrt{\alpha_{2}}\right) .
$$

In order to avoid delay-free loops [1] and maintain the number of delays to the minimum of two, the constraints

$$
A=z^{-1}, \quad \text { and } \quad B \text { or } D=z^{-1}
$$

must be satisfied. Therefore, two cases are possible, as follows:

Case I: $A=B=z^{-1}$

Case II: $A=D=z^{-1}$

Case I

For Case I, the characteristic polynomial of (1) becomes

$$
D(z)=z^{2}-z\left(C+D+m_{1}\right)+C D+m_{1} D+m_{2}+E .
$$

In order to obtain low se.ısitivity, multipliers $C, D$, and $E$ must be chosen as

$$
\begin{aligned}
C+D & =\operatorname{Int}\left[-\alpha_{1}\right] \\
E & =\operatorname{Int}\left[\alpha_{2}+\alpha_{1} D+D^{2}\right]
\end{aligned}
$$

where Int. $[x]$ is the closest integer to $x$. Equations (4) and (5) force the values of $m_{1}$ and $m_{2}$ to be low and, as demonstrated in [9], this assures low sensitivity of the

\begin{tabular}{|c|c|c|c|c|}
\hline \multirow{2}{*}{ STRUCTURE } & \multicolumn{3}{|c|}{ COEFFICIENTS } & \multirow{2}{*}{ RANGE OF $\alpha_{1}$} \\
\hline & 0 & C & $E$ & \\
\hline$I-1$ & 1 & 1 & 0 & \multirow{2}{*}{$-2.0<a_{1}<-1.5$} \\
\hline I-2 & 0 & 2 & 1 & \\
\hline$I-3$ & 2 & 0 & 1 & $-2.0<\alpha_{1}<-1.75$ \\
\hline$I-4$ & 2 & 0 & 2 & $-1.75<\alpha_{1}<-1.5$ \\
\hline$I-5$ & 0 & 1 & 1 & \multirow{2}{*}{$-1.5<\alpha_{1}<-0.5$} \\
\hline$I-6$ & 1 & 0 & 1 & \\
\hline$I-7$ & 0 & 0 & 1 & \multirow{3}{*}{$-0.5<\alpha_{1}<0.5$} \\
\hline$I-8$ & 1 & -1 & 2 & \\
\hline $1-9$ & -1 & 1 & 2 & \\
\hline $1-10$ & 0 & -1 & 1 & \multirow{2}{*}{$0.5<\alpha_{1}<1.5$} \\
\hline I-11 & -1 & 0 & 1 & \\
\hline $1-12$ & -2 & 0 & 2 & $1.5<\alpha_{1}<1.75$ \\
\hline$I-13$ & 0 & -2 & 1 & \multirow{2}{*}{$1.5<\alpha_{1}<2.0$} \\
\hline$I-14$ & -1 & -1 & 0 & \\
\hline$I-15$ & -2 & 0 & 1 & $1.75<\alpha_{1}<2.0$ \\
\hline
\end{tabular}
transfer function with respect to changes in $m_{1}$ and $m_{2}$ for the case where floating-point arithmetic is used or for the case where fixed-point arithmetic is used but the coefficients are stored in normalized floating-point form.

The choice of structure tends to depend heavily on the pole positions. Therefore, several possibilities must be ex-
TABLE I

Structures for CASE I

amined. If the poles are close to $z=1$, then $\alpha_{1} \cong-2$ and $\alpha_{2} \cong 1$, and so

$$
C+D=2
$$

We can thus assign

$$
C=1, \quad D=1 \quad \text { and } \quad E=0
$$

This choice of coefficients yields a structure due to Agarwal and Burrus [9], which is suitable for values of $\alpha_{1}$ in the range $-2.0<\alpha_{1}<-1.5$. It will be referred to as structure I-1. Alternatively, if

$$
C=2, \quad D=0 \quad \text { and } \quad E=1
$$

another structure due to the same authors [9] is obtained. This structure is suitable for the same range of $\alpha_{1}$ as structure I-1 and will be referred to as structure I-2. A third possibility is obtained by letting

$$
C=0, \quad D=2 \quad \text { and } \quad E=1
$$

This structure will be referred to as structure I-3, and is to our knowledge new. It is suitable for values of $\alpha_{1}$ in the range $-2.0<\alpha_{1}<-1.75$.

By considering poles which are close to the unit circle $|z|=1$, several new structures can be obtained for different ranges of values of $\alpha_{1}$, as shown in Table $I$.

Case II

For Case II, the characteristic polynomial of (1) becomes

$$
D(z)=z^{2}-z\left(B+C+m_{1}-m_{2} B-B E\right)+B C+m_{1} B .
$$

In order to obtain low sensitivity, multiplier coefficients $B$, 


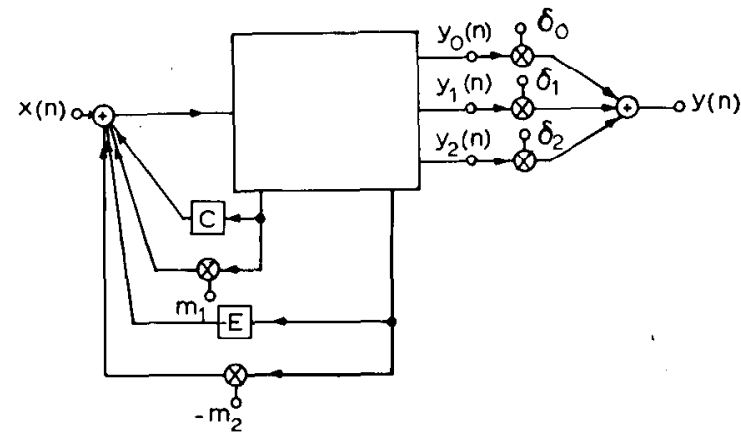

Fig. 3. Zero-placement technique.

TABLE II

STRUCTURES FOR CASE II

\begin{tabular}{|c|c|c|c|c|}
\hline \multirow{2}{*}{ STRUCTURE } & \multicolumn{3}{|c|}{ COEFFICIENTS } & \multirow{2}{*}{ RANGE Of $\alpha_{1}$} \\
\hline & B & $\mathrm{C}$ & $E$ & \\
\hline II-1 & 1 & 1 & 0 & $-2.0<\alpha_{1}<-1.5$ \\
\hline II -2 & 1 & 1 & 1 & $-1.5<\alpha_{1}<-0.5$ \\
\hline II -3 & 1 & 1 & 2 & $-0.5<\alpha_{1}<0$ \\
\hline II -4 & -1 & -1 & 2 & $0<\alpha_{1}<0.5$ \\
\hline II -5 & -1 & -1 & 1 & $0.5<\alpha_{1}<1.5$ \\
\hline II -6 & -1 & -1 & 0 & $1.5<\alpha_{1}<2.0$ \\
\hline
\end{tabular}

$C$, and $E$ must be chosen as

$$
\begin{aligned}
& B=1, \quad C=1 \\
& E=\operatorname{Int} .\left[\alpha_{1}+\alpha_{2}+1\right]
\end{aligned}
$$

for poles with positive real part, and

$$
\begin{aligned}
& B=-1, \quad C=-1 \\
& E=- \text { Int. }\left[\alpha_{1}-\alpha_{2}-1\right]
\end{aligned}
$$

for poles with negative real part.

If the poles are close to $z=1$, we can assign

$$
B=1, C=1, \text { and } E=0 \text {. }
$$

This choice of coefficients yields a structure due to Nishimura, Hirano, and Pal [10], which is suitable for values of $\alpha_{1}$ in the range $-2.0<\alpha_{1}<-1.5$. It will be referred to as structure II-1.

As for Case I, several new structures can be generated by considering poles which are close to the unit circle. These are summarized in Table II.

\section{Zero Placement}

The general second-order transfer function

$$
H(z)=\frac{Y(z)}{X(z)}=\frac{\gamma_{0} z^{2}+\gamma_{1} z+\gamma_{2}}{z^{2}+\alpha_{1} z+\alpha_{2}}
$$

can be realized by modifying the low-sensitivity sections generated so far, as depicted in Fig. 3. The design equations required to complete a design can readily be derived, as shown in Table III.

An alternative possibility is to take signal $y_{0}(\mathrm{~m})$ just after the input adder, as shown in Fig. 4. In this case, the design equations for the feedforward multipliers are given in Table IV.

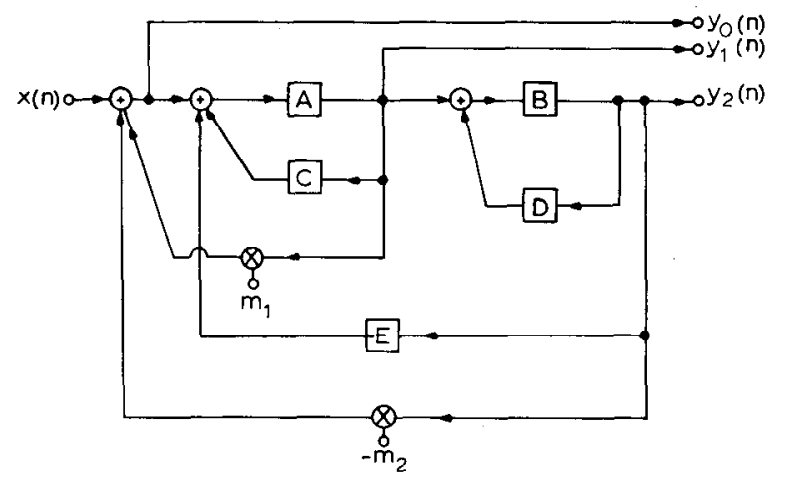

Fig. 4. Alternative zero-placement technique.

TABLF III

EQUaTIONS For THE DESIGN OF BIQUadRaTIC TRANSFER FUNCTIONS

\begin{tabular}{|c|c|c|c|c|c|}
\hline Case & $\delta_{0}$ & $\delta_{1}$ & $\delta_{2}$ & $m_{1}$ & $m_{2}$ \\
\hline I & $\gamma_{0}$ & $\gamma_{1}+D \gamma_{0}$ & $r_{2}+D \gamma_{1}+D^{2} \gamma_{0}$ & $-\alpha_{1}-C-D$ & $\alpha_{2}+\alpha_{1} D+D^{2}-E$ \\
\hline II & $r_{0}$ & $-\frac{\gamma_{2}}{B}$ & $r_{0}+\frac{\gamma_{1}}{B}+\frac{\gamma_{2}}{B^{2}}$ & $\frac{\alpha_{2}}{B}-C$ & $1+\frac{\alpha_{1}}{B}+\frac{\alpha_{2}}{B}-E$ \\
\hline
\end{tabular}

TABLE IV

Alternative Equations for the Design of Biquadratic Transfer Functions

\begin{tabular}{|c|c|c|c|}
\hline Case & $\delta_{0}$ & $\delta_{1}$ & $\delta_{2}$ \\
\hline$I$ & $\gamma_{0}$ & $\gamma_{1}{ }^{+} \gamma_{0} \mathrm{C}+\gamma_{0} D$ & $\gamma_{2}{ }^{+} \gamma_{1} \mathrm{D}-\gamma_{0} \mathrm{E}+\gamma_{0} \mathrm{D}^{2}$ \\
\hline $\mathrm{II}$ & $\gamma_{0}$ & $\gamma_{0} \mathrm{C}-\frac{\gamma_{2}}{\mathrm{~B}}$ & $\frac{\gamma_{2}}{\mathrm{~B}^{2}}+\frac{\gamma_{1}}{\mathrm{~B}}-\gamma_{0} \mathrm{E}+\gamma_{0}$ \\
\hline
\end{tabular}

The proposed structures can be implemented in terms of ROM-accumulator arithmetic [12], [13] or in terms of stored-product arithmetic [14]. Furthermore, the internal scaling strategies described in [9], [10], and [13] can be used as an alternative to ESS.

\section{SEnSITIVity Analysis}

The sensitivity of the transfer function

$$
H(z)=\frac{N(z)}{D(z)}
$$

of a digital-filter structure with respect to variations in multiplier constant $m_{i}$ is defined as

$$
S_{m_{i}}^{H(z)}=\frac{N_{m_{i}}(z)}{D_{m_{i}}(z)}=\frac{m_{i}}{H(z)} \frac{\partial H(z)}{\partial m_{i}} .
$$

If

the function

$$
H\left(e^{j \omega T}\right)=M(\omega) e^{j \theta(\omega)}
$$

$$
S=\sum_{i=1}^{k}\left|S_{m_{i}}^{H\left(e^{j \omega T}\right)}\right|
$$

can be formed where $k$ is the number of multipliers in the structure and

$$
\left|S_{m_{i}}^{H\left(e^{j \omega T}\right)}\right|=\left\{\left(S_{m_{i}}^{M(\omega)}\right)^{2}+\left|m_{i}\right|^{2}\left(\frac{\partial \theta(\omega)}{\partial m_{i}}\right)^{2}\right\}^{1 / 2} .
$$

If the poles of the structure are close to the unit circle $|z|=1$, and $m_{i}$ is a noninteger denominator multiplier 
TABLE V

Numerator and Denominator Polynomials of Sensitivities

\begin{tabular}{|c|c|c|c|}
\hline Case & $N_{m_{1}}(z)$ & $N_{m_{2}}(z)$ & $D_{m_{1}}(z)$ \\
\hline$I$ & $m_{1}(z-D)$ & $-m_{2}$ & $D(z)$ \\
\hline$I I$ & $m_{1}(z-B)$ & $-m_{2} B z$ & $D(z)$ \\
\hline
\end{tabular}

constant, then for $\omega$ close to the pole frequency $\omega_{0}$ (see (1))

$$
\left|S_{m_{i}}^{M(\omega)}\right| \gg\left|m_{i}\right|\left|\frac{\partial \theta(\omega)}{\partial m_{i}}\right|
$$

and hence

$$
\left|S_{m_{i}}^{H\left(e^{j \omega T}\right)}\right| \approx\left|S_{m_{i}}^{M(\omega)}\right| \text {. }
$$

In addition, if $m_{j}$ is a numerator multiplier constant then

$$
\left|S_{m_{i}}^{H\left(e^{j \omega T}\right)}\right| \gg\left|S_{m_{j}}^{H\left({ }^{j \omega T}\right)}\right|
$$

and, therefore, for $\omega \simeq \omega_{0}$ (13)-(15) yield

$$
S \simeq \sum_{i=1}^{k_{d}}\left|S_{m_{i}}^{H\left(e^{j \omega T}\right)}\right| \sim \sum_{i=1}^{k_{d}}\left|S_{m_{i}}^{M(\omega)}\right|
$$

where $k_{d}$ is the number of denominator noninteger multiplier constants. This quantity can serve as a sensitivity measure which can be used for the comparison of different high-selectivity structures.

As was demonstrated in Section II, several structures are possible in which ESS can efficiently be applied. Furthermore, for each permissible range of $\alpha_{1}$ at least two distinct structures are possible, as can be seen in Tables I and II. It would thus be a useful exercise to identify an optimal subset of these structures.

The value of $S$ at a specific frequency and its average value over the baseband are strongly related to the maximum value of $S$, denoted by $\hat{S}$, which occurs at $\omega=\omega_{0}$. It is thus possible to identify the optimal structures in Tables I and II by using $\hat{S}$ as a sensitivity measure.

For $-1.5 \leqslant \alpha_{1}<-0.5$, the structures to be compared are I-5, I- $\iota$, and II-2. Assuming that $\alpha_{2}$ is close to unity, (12) and (16), and Table $\mathrm{V}$ yield the maximum sensitivities of these structures as

$$
\begin{gathered}
\hat{S}_{\mathrm{I}-\mathrm{S}} \simeq \frac{N_{\mathrm{I}-5}}{\left|D\left(e^{j \omega_{0} T}\right)\right|} \\
\hat{S}_{\mathrm{I}-6} \simeq \frac{N_{\mathrm{I}-6}}{\left|D\left(e^{j \omega_{0} T}\right)\right|} \\
\hat{S}_{\mathrm{II}-2} \simeq \frac{N_{\mathrm{II}-2}}{\left|D\left(e^{j \omega_{0} T}\right)\right|}
\end{gathered}
$$

where

$$
\begin{gathered}
N_{\mathrm{I}-5}=1-\alpha_{2}+\left|1+\alpha_{1}\right| \\
N_{\mathrm{I}-6}=\left|\alpha_{1}+\alpha_{2}\right|+\left|1+\alpha_{1}\right| \sqrt{\left(2-2 \cos \omega_{0}\right)} \\
N_{\mathrm{II}-2}=\left|\alpha_{1}+\alpha_{2}\right|+\left(1-\alpha_{2}\right) \sqrt{\left(2-2 \cos \omega_{0}\right)} . \\
\text { For }-1.5 \leqslant \alpha_{1}<-1 \text { and } \alpha_{2}<1 \\
N_{\mathrm{l}-5}=-\left(\alpha_{1}+\alpha_{2}\right)
\end{gathered}
$$

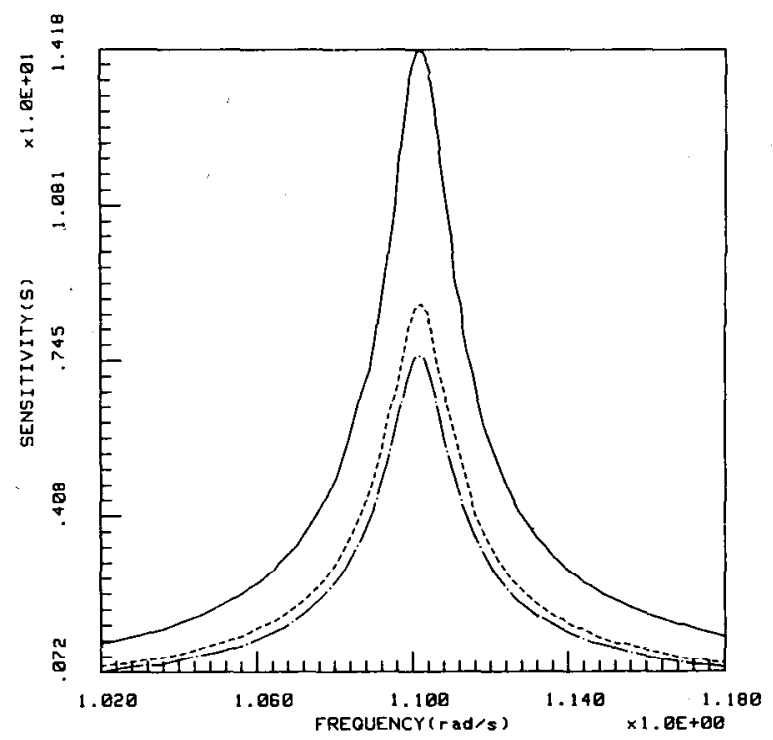

Fig. 5. Sensitivity versus frequency: - Structure $1-6, \ldots-$ Structure I-5, - $\cdot-\cdots$ Structure II- 2 .

$$
\begin{aligned}
& N_{\mathrm{I}-6}=-\left(\alpha_{1}+\alpha_{2}\right)-\left(1+\alpha_{1}\right) \sqrt{\left(2-2 \cos \omega_{0}\right)} \\
& N_{\mathrm{II}-2}=-\left(\alpha_{1}+\alpha_{2}\right)+\left(1-\alpha_{2}\right) \sqrt{\left(2-2 \cos \omega_{0}\right)}
\end{aligned}
$$

and hence

$$
\hat{S}_{\mathrm{I}-5}<\min \left\{\hat{S}_{\mathrm{I}-6}, \hat{S}_{\mathrm{II}-2}\right\} .
$$

$$
\text { For } \begin{aligned}
-1 & \leqslant \alpha_{1}<-\alpha_{2}, \\
N_{\mathrm{I}-5} & =2-\alpha_{2}+\alpha_{1} \\
N_{\mathrm{I}-6} & =-\left(\alpha_{1}+\alpha_{2}\right)+\left(1+\alpha_{1}\right) \sqrt{\left(2-2 \cos \omega_{0}\right)} \\
N_{\mathrm{II}-2} & =-\left(\alpha_{1}+\alpha_{2}\right)+\left(1-\alpha_{2}\right) \sqrt{\left(2-\cos \omega_{0}\right)} .
\end{aligned}
$$

Evidently,

$$
N_{\text {I- } 6}<N_{\text {II-2 }}
$$

and, in addition

$$
N_{\mathrm{I}-6}<N_{\mathrm{I}-5}
$$

as can be shown by adding $\left(\alpha_{1}+\alpha_{2}\right)$ to each of (17) and (18), and then dividing each by $\left(1+\alpha_{1}\right)$. As a consequence

$$
\hat{S}_{\mathrm{I}-6}<\min \left\{\hat{S}_{\mathrm{I}-5}, \hat{S}_{\mathrm{II}-2}\right\} \text {. }
$$

Similarly, for $-\alpha_{2} \leqslant \alpha_{1} \leqslant-0.5$

$$
\begin{aligned}
& N_{\mathrm{I}-5}=2-\alpha_{2}+\alpha_{1} \\
& N_{\mathrm{I}-6}=\left(\alpha_{1}+\alpha_{2}\right)+\left(1-\alpha_{1}\right) \sqrt{\left(2-2 \cos \omega_{0}\right)} \\
& N_{\mathrm{II}-2}=\left(\alpha_{1}+\alpha_{2}\right)+\left(1-\alpha_{2}\right) \sqrt{\left(2-2 \cos \omega_{0}\right)}
\end{aligned}
$$

and as above we can show that

$$
\hat{S}_{\mathrm{II}-2}<\min \left\{\hat{S}_{\mathrm{I}-5}, \hat{S}_{\mathrm{I}-6}\right\} .
$$

In effect, the choice of structure tends to depend heavily on the relative values of coefficients $\alpha_{1}$ and $\alpha_{2}$. For example, if $\alpha_{1}=-0.9$ and $\alpha_{2}=0.995$, I-5, I-6, and II-2 are possible structures, and according to (20) the optimal one is II-2. This result is confirmed by the sensitivity plots in Fig. 5. 


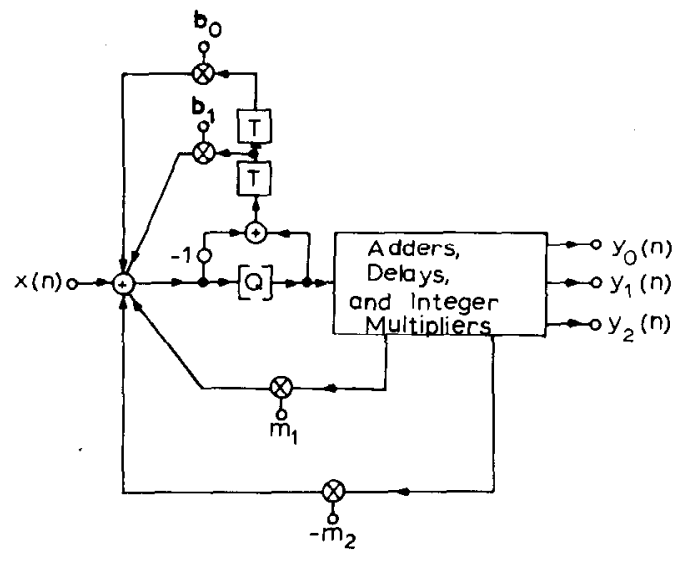

Fig. 6. Application of ESS.

TABLE VI

OPTIMUM STRUCTURES

\begin{tabular}{|l|c|c|}
\hline Range of $\alpha_{1}$ & Structure & Condition for 0ptimality \\
\hline$-2<\alpha_{1}<-1.5$ & $I I-1$ & $-2<\alpha_{1}<-1.5$ \\
\hline \multirow{3}{*}{$-1.5<\alpha_{1}<-0.5$} & $1-5$ & $-1.5<\alpha_{1}<-1.0$ \\
\cline { 2 - 3 } & $I-6$ & $-1.0<\alpha_{1}<-\alpha_{2}$ \\
\cline { 2 - 3 } & $1 I-2$ & $-\alpha_{2}<\alpha_{1}<-0.5$ \\
\hline$-0.5<\alpha_{1}<0.5$ & $I-7$ & $-0.5<\alpha_{1}<0.5$ \\
\hline & $I-10$ & $1.0<\alpha_{1}<1.5$ \\
\cline { 2 - 3 } $0.5<\alpha_{1}<1.5$ & $1-11$ & $\alpha_{2}<\alpha_{1}<1.0$ \\
\cline { 2 - 3 } & $I I-5$ & $0.5<\alpha_{1}<\alpha_{2}$ \\
\hline $1.5<\alpha_{1}<2$ & $I I-6$ & $1.5<\alpha_{1}<2$ \\
\hline
\end{tabular}

The above approach has been applied for the remaining ranges of $\alpha_{1}$. The optimum structures identified are summarized in Table VI.

It should be mentioned that the sensitivity analysis of this section, like that reported in [9], is more appropriate for digital-filter structures implemented in terms of floating-point arithmetic or in terms of fixed-point arithmetic but with the coefficients stored in normalized floating-point form. Nevertheless, the experimental results presented in Section VI show that the conclusions reached also hold in the case where standard fixed-point arithmetic is used in conjunction with signal scaling.

\section{ApPlication of ESS}

ESS can be applied in the structures of Figs. 2 to 3 by including a quantizer $Q$ and an appropriate substructure at the output of the input adder, as illustrated in Fig. 6. In this way, the power-spectral density of the output noise can properly be shaped. In effect, error feedback is applied, which can be adjusted to force zeros in the power-spectral density of the output noise and by choosing coefficients $b_{0}$ and $b_{1}$ using the formulas in [8], the output noise can be reduced or minimized.

The application of ESS requires double-precision arithmetic for the input adder as well as for multipliers $m_{1}$ and $m_{2}$. However, single-precision arithmetic is entirely satisfactory for ESS multipliers $b_{0}$ and $b_{1}$. It should be men-
TABLE VII

Filter SPECIFICATIONS

\begin{tabular}{|c|c|c|c|c|}
\hline Parameter & $\begin{array}{l}\text { Elliptic } \\
\text { Lowpass } \\
\text { Filter }\end{array}$ & $\begin{array}{l}\text { Chebyshev } \\
\text { Highpass } \\
\text { Ftlter }\end{array}$ & $\begin{array}{l}\text { Elifptic } \\
\text { Bandpass } \\
\text { Filter }\end{array}$ & $\begin{array}{l}\text { Butterworth } \\
\text { Bands top } \\
\text { Filter }\end{array}$ \\
\hline$A_{p}, \mathrm{~dB}$ & 1.0 & 0.6 & 0.5 & 2.0 \\
\hline $\mathrm{A}_{\mathrm{a}}, \mathrm{dB}$ & 72.9 & 48.6 & 66.2 & 33.9 \\
\hline$\omega_{\mathrm{p} 1}, \mathrm{rad} / \mathrm{s}$ & 250.0 & 4000.0 & 1180.0 & 2300.0 \\
\hline$\omega_{\mathrm{p} 2}, \mathrm{rad} / \mathrm{s}$ & - & - & 1220.0 & 2700.0 \\
\hline$\omega_{a 1}, \mathrm{rad} / \mathrm{s}$ & 400.0 & 3300.0 & 1050.0 & 2450.0 \\
\hline$\omega_{\mathrm{a} 2}, \mathrm{rad} / \mathrm{s}$ & - & & 1350.0 & 2550.0 \\
\hline$\omega_{\mathrm{s}}, \mathrm{rad} / \mathrm{s}$ & $10,000.0$ & & \\
\hline
\end{tabular}

tioned that the precision of coefficients $b_{0}$ and $b_{1}$ can be reduced and sometimes $b_{0}$ and $b_{1}$ can even be chosen to be powers of two so as to replace the corresponding multiplications by data shifts. This reduces the complexity of the structure but our ability to reduce or minimize output noise is diminished.

\section{COMPARISON}

For the sake of comparison, the cascade approach was used to design four 6th-order filters which included an elliptic low pass, a Chebyshev high pass, an elliptic bandpass, and a Butterworth bandstop filter. The specifications of the various filters are given in Table VII where

$$
\begin{array}{ll}
A_{p} & \text { Passband ripple, } \mathrm{dB} \\
A_{a} & \text { Minimum stopband attenuation, dB } \\
\omega_{p 1}, \omega_{p 2} & \text { Passband edges, } \mathrm{rad} / \mathrm{s} \\
\omega_{a 1}, \omega_{a 2} & \text { Stopband edges, } \mathrm{rad} / \mathrm{s} \\
\omega_{s} & \text { sampling frequency, } \mathrm{rad} / \mathrm{s} .
\end{array}
$$

Designs were obtained with the proposed structures [1], with the direct canonic structure, and with the section-optimal structure described in [15]. Signal scaling, based on the $L_{\infty}$ norm, was applied in all designs, and the section ordering was chosen to minimize the output noise in each case. The implementation was assumed to be in terms of fixed-point arithmetic, and quantization of coefficients and products was assumed to be by rounding. The various designs were compared on the basis of actual amplitude responses with the coefficients quantized, and on the basis of computed output-noise spectra.

The results of the sensitivity analysis are depicted in Fig. 7(a)-(d). As can be seen, for the lowpass filter the sectionoptimal structure leads to better results than the proposed structure; for the high-pass and bandpass filters, the best results are achieved by using proposed structures II- 6 and I-5, respectively; and for the bandstop filter the best results are achieved by using either proposed structure I-7 or the canonic structure. The most sensitive structure is the canonic structure for the low-pass and high-pass filters, and the section-optimal structure for the bandpass and bandstop filters.

The roundoff-noise analysis involved the computation of the relative power spectral density (RPSD) [1] in the various filter designs. In designs based on the proposed struc- 


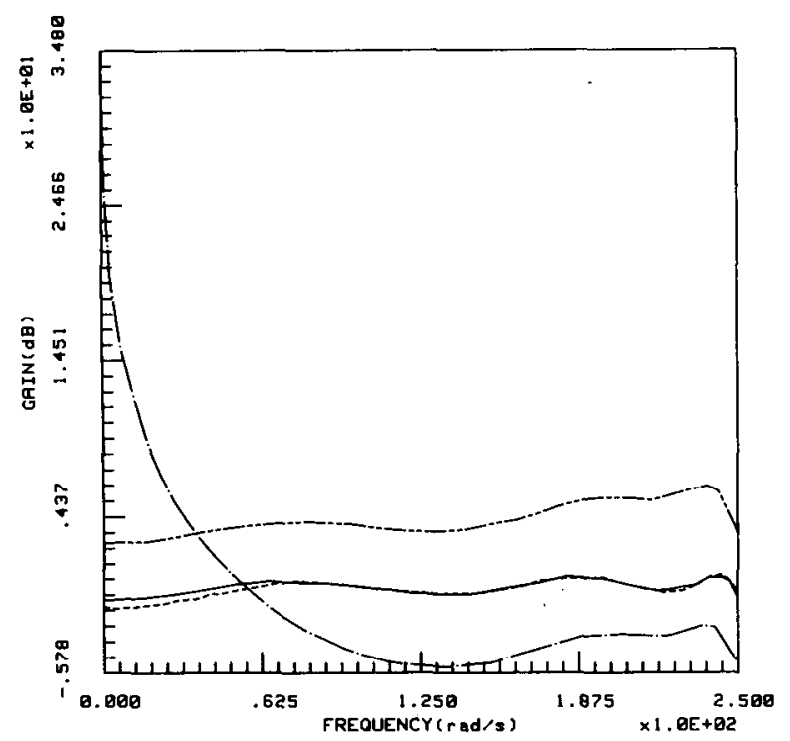

(a)

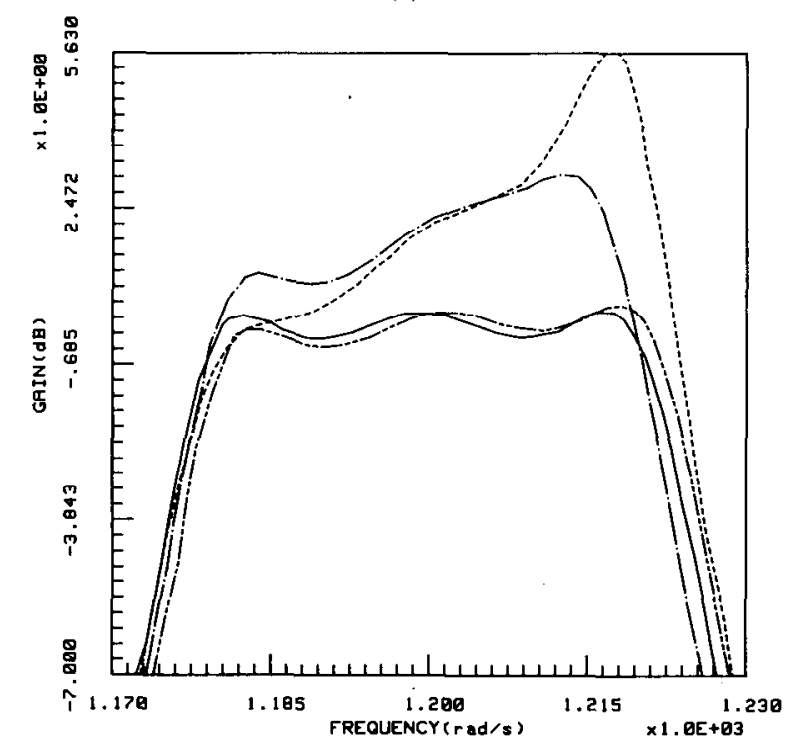

(c)

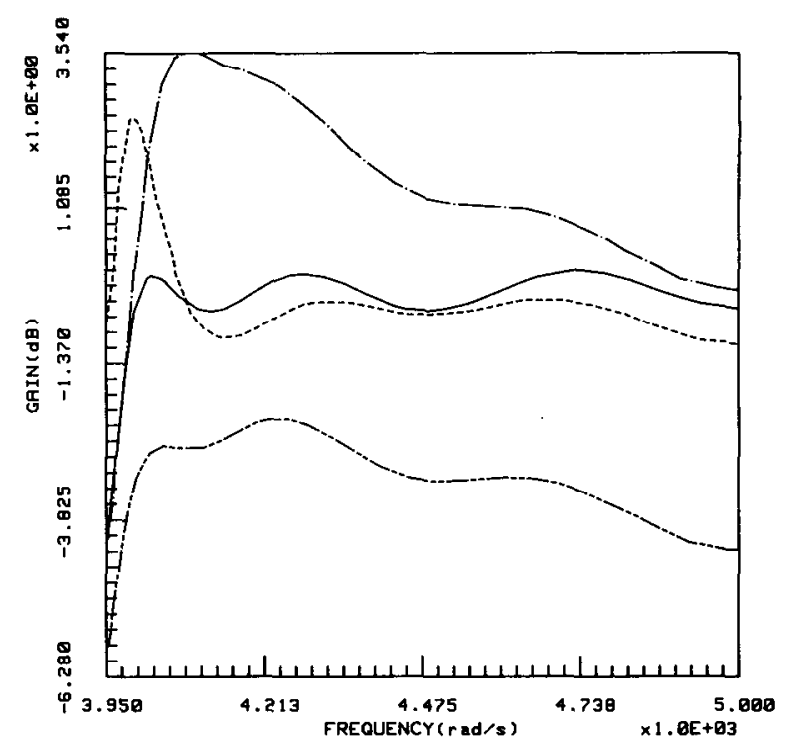

(b)

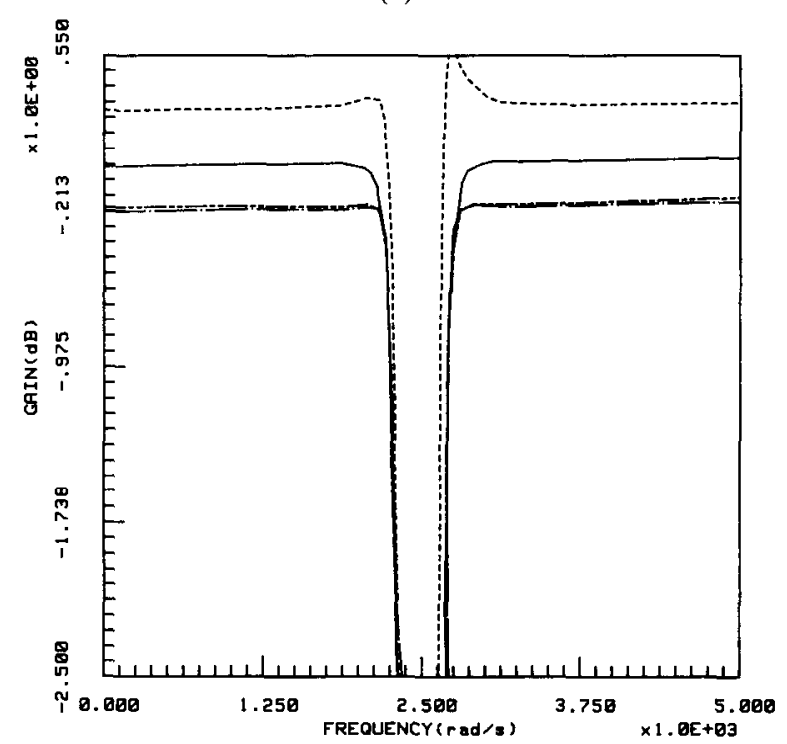

(d)

Fig. 7. Amplitude responses. (a) Low-pass filter, 8 bits. (b) High-pass filter, 5 hits. (c) Bandpass filter, 7 bits. (d) Bandstop filter, 5 bits. - Ideal amplitude response, -...- Section-optimal structure, - - - - Direct-canonic structure, - - - - New structure (II-1 for lowpass filter, II-6 for high-pass filter, I-5 for bandpass filter, I-7 for bandstop filter).

tures, ESS has been applied using the formulas in [8]. The quantization of products was carried out after the adders for all sections in the cascade except for the numerator multipliers of the output section where the quantization was carried out before the adder. ESS has not been applied in the corresponding designs based on the section-optimal structure since the number of multiplications would then become excessive. It should be mentioned that the proposed structures with ESS incorporated require between five and seven multipliers per section whereas the sectionoptimal structure without ESS requires nine multipliers per section.

The results of the roundoff-noise analysis are depicted in Fig. 8(a)-(d). As can be seen, for the low-pass, high-pass, and bandpass filters, the proposed structures with ESS incorporated lead to superior results, in particular, if sec- ond-order ESS is applied. For the bandstop filter, on the other hand, the passband average of the RPSD is lower in the section-optimal structure whereas the passband maximum of the RPSD is lower in the proposed structure. It should be mentioned that a similar reduction in output roundoff noise can be achieved by applying ESS to direct canonic structures. However, as was pointed out earlier, these structures are almost always more sensitive relative to the proposed structures.

\section{CONCLUSIONS}

A systematic and exhaustive procedure has been described for the generation of economical low-sensitivity digital-filter structures which are amenable to the application of ESS. The procedure has then been used to generate the structures of Tables I and II. 


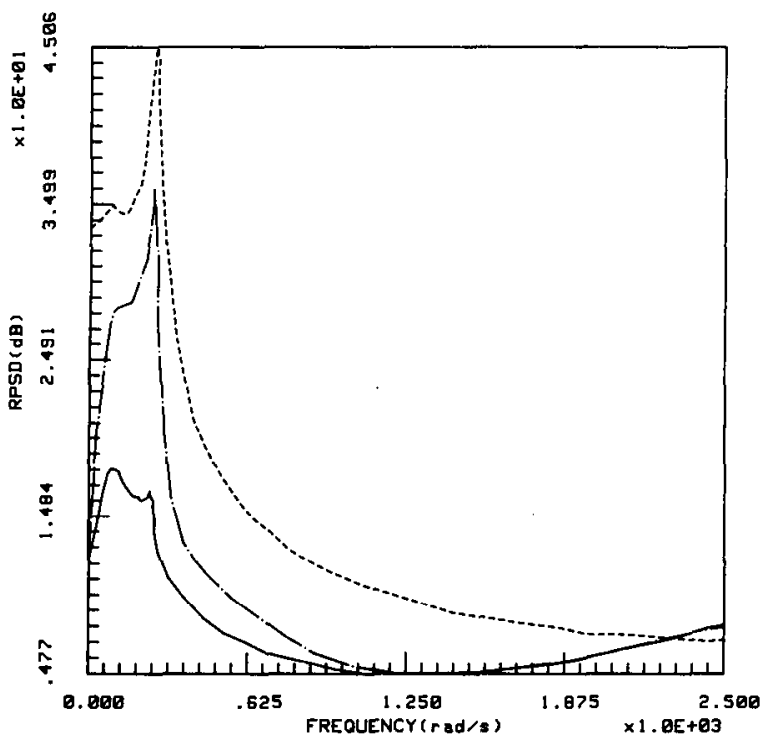

(a)

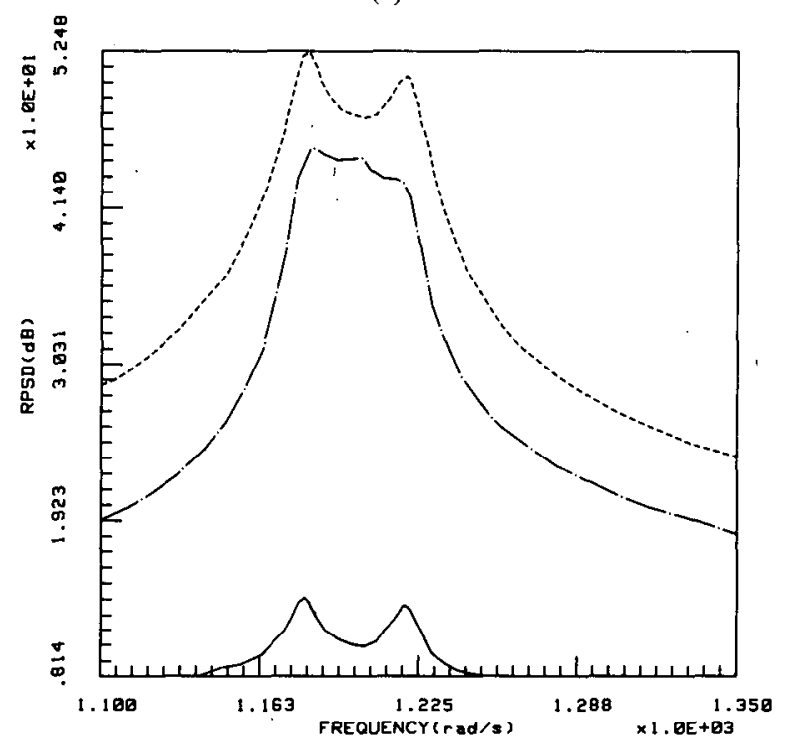

(c)

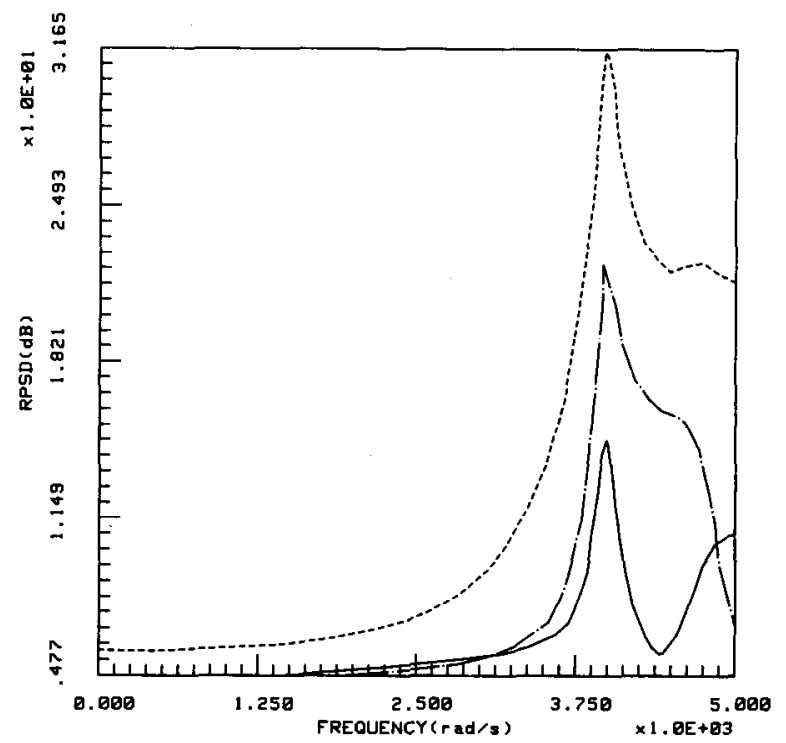

(b)

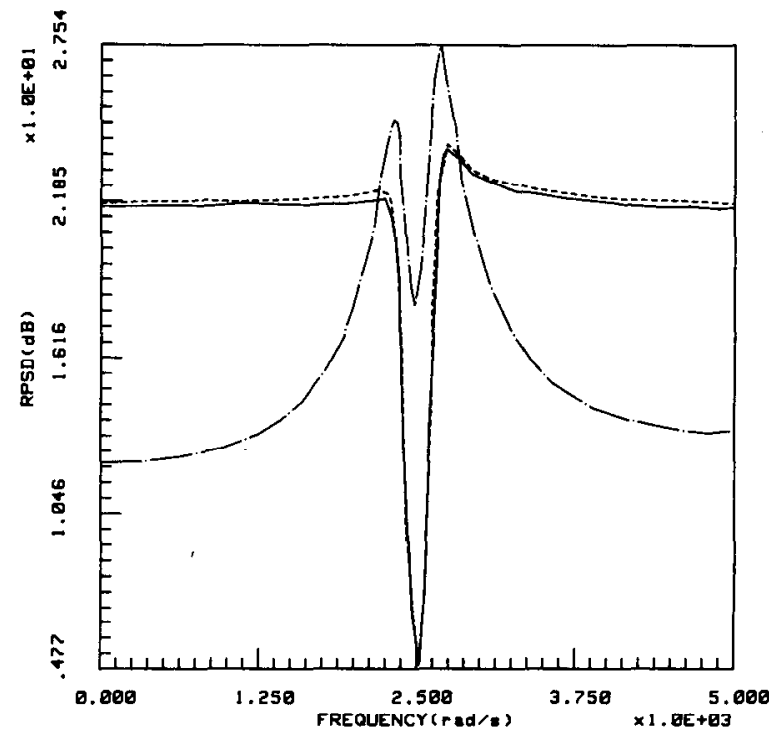

(d)

Fig. 8. Output-noise spectra. (a) Low-pass filter. (b) High-pass filter. (c) Bandpass filter. (d) Bandstop filter: -.... Section-optimal structure, - - - - - New structure with 1st-order ESS, __ New structure with 2nd-order ESS (II-1 for low-pass filter, II-6 for high-pass filter, I-5 for bandpass filter, I-7 for bandstop filter).

In all, 21 structures were obtained and except for structures I-1, I-2, and II-1, which were reported in [9], [10], they are thought to be new. For each range of $\alpha_{1}$, at least two distinct structures are possible but, through the sensitivity analysis of Section IV, the optimum structure for the transfer function under consideration can easily be chosen, as shown in Table VI.

A sensitivity comparison has shown that the proposed structures are usually superior relative to corresponding direct canonic structures and also relative to the sectionoptimal structure.

A roundoff noise comparison has shown the proposed structures with ESS incorporated to be superior relative to the section-optimal structure, except in the case of bandstop filters where a lower passband average of the RPSD can be achieved by using the section-optimal structure.
Further, despite the additional multipliers needed for ESS, these structures require fewer multipliers per second-order section.

\section{ACKNOWLEDGMFNT}

The authors are grateful to Coordenação do Aperfeiçoamento de Pessoal de Nivel Superior, Brazil, and to the Natural Sciences and Engineering Research Council, Canada for supporting this research.

\section{REFERENCES}

[1] A. Antoniou, Digital Filters: Analysis and Design. New York: McGraw-Hill, 1979.

[2] A. Fettweis, "Digital filter structures related to classical filter networks," Arch. Elek. Übertragung., vol. 25, pp. 79-89, Feb. 1971.

[3] A. Antoniou and M. G. Rezk, "A comparison of cascade and wave fixed-point digital filter structures," IEEE Trans. Circuits Syst., vol. CAS-27, pp. 1184-1194, Dec. 1980. 
[4] T. Thong and B. Liu, "Error spectrum shaping in narrowband recursive digital filters," IEEE Trans. Acoust., Speech, Signal Processing, vol. ASSP-25, pp. 200-203, Apr. 1977.

[5] T. L. Chang, "Error spectrum shaping structures for digital filters," in Proc. 13th Asilomar Conf. Circuits Syst. Comput., Pacific Grove, CA, pp. 279-283, Nov. 1979 .

[6] D. C. Munson and B. Liu, "Narrow-band recursive filters with error spectrum shaping," IEEE Trans. Circuits Syst., vol. CAS-28, pp. $160-163$, Feb. 1981 .

[7] W. E. Higgins and D. C. Munson, "Noise reduction strategies for digital filters: Error spectrum shaping versus the optimal linear state-space formulation," IEEE Trans. Acoust., Speech, Signal Processing, vol. ASSP-30, pp. 963-973, Dec. 1982

[8] ' "Optimal and suboptimal error-spectrum shaping for cascade-form digital filters," IEEE Trans. Circuits Syst., vol. CAS31, pp. 429-437, May 1984

[9] R. C. Agarwal and C. S. Burrus, "New recursive digital filter structures having very low sensitivity and roundoff noise," IEEE Trans. Circuits Syst., vol. CAS-22, pp. 921-927, Dec. 1975.

[10] S. Nishimura, K. Hirano, and R. N. Pal, "A new class of very low sensitivity and low roundoff noise recursive digital filter structures," IEEE Trans. Circuits Syst., vol. CAS-28, pp. 1152-1158, Dec. 1981.

[11] K. Haug and E. Luder, "Determination of all equivalent and canonic second order digital filter structures," Arch. Elek. Ubertragung., vol. 36, pp. 436-442, Nov.-Dec. 1982.

[12] D. C. Munson and B. Liu, "ROM/ACC realization of digital filters for poles near the unit circle," IEEE Trans. Circuits Syst., vol. CAS-27, pp. 147-151, Feb. 1982.

[13] "Low-noise realization for narrow-band recursive digital filters," IEEE Trans. Acoust., Speech, Signal Processing, vol. ASSP-28, pp. 41-54, Feb. 1980.

[14] O. Monkewich and W. Steenaart, "Stored product digital filtering with nonlinear quantization," in Proc. IEEE Int. Symp. Circuits Syst., pp. 157-160, 1976.

[15] L. B. Jackson, A. G. Lindgren, and Y. Kim, "Optimal synthesis of second-order state-space structures for digital filters," IEEE Trans. Circuits Syst., vol. CAS-26, pp. 149-153, Mar. 1979.

\section{承}

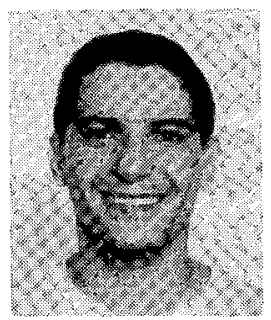

Paulo S. R. Diniz ('s'80-M'84) was born in Niteroi, Brazil. He received the B.Sc. degree from Universidade Federal do Rio de Janeiro (UFRJ), in 1978, the M.Sc. degree from COPPE/UFRJ in 1981 , and the Ph.D. degree from Concordia University, Montreal, Canada, in 1984, all in electrical engineering.

Since 1979, he has been with the Departmen of Electronic Engineering, UFRJ. He has also been with the Department of Electrical Engineering of COPPE/UFRJ, since 1984, where he is associate professor. His teaching and research interests include passive and active network synthesis, electronic circuits, digital filters and digital signal processing.

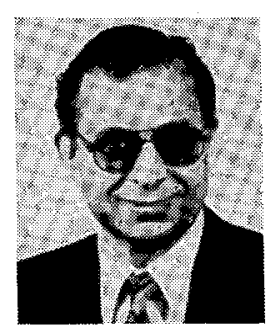

Andreas Antoniou (M'69-SM'79-F'82) received the B.Sc:(Eng.) and Ph.D degrees in Electrical Engineering from London University in 1963 and 1966, respectively.

From 1966 to 1969 he was Senior Scientific Officer at the Post Office Research Department, London, England, and from 1969 to 1970, he was a member of the Scientific Staff at the R\&D Laboratories of Northern Electric Company Ltd., Ottawa, Ontario, Canada. From 1970 to 1983 he served in the Department of Electrical Engineering, Concordia University, Montreal, Quebec, Canada, as Professor from June 1973 and as Chairman from December 1977. On July 1, 1983 he was appointed founding Chairman of the Department of Electrical Engineering, University of Victoria, Victoria, B.C., Canada.

His teaching and research interests are in the areas of electronics, network synthesis, digital system design, active and digital filters, and digital signal processing. He has published a number of papers on electronic circuits, active filters, and digital filters. He has authored Digital Filters: Analysis and Design, (New York: McGraw-Hill). One of his papers on gyrator circuits was awarded the Ambrose Fleming Premium by the Institution of Electrical Engineers, UK.

Dr. Antoniou is a Member of the Order of Engincers of Qucbce, and a Fellow of the Institution of Electrical Engineers. He was Associate Editor for IEEE Transactions on CircutTs and Systems during the period June 1983 to May 1985. He is now serving as Editor of the same TRANSACTIONS. 